\title{
Ad-Hoc Reviewers from 2011
}

The Journal of Nonverbal Behavior greatly appreciates the assistance of the Editorial Board as well as the following ad hoc manuscript reviewers:

Reginald Adams Jr.

Anthony Atikinson

Adrian Bangerter

Janet Bavelas

Andrew Bayliss

Vaughn Becker

Lane Beckes

Joanna Blake

R. Thomas Boone

Amanda Brown

Peter Bull

Dana Carney

Laurence Conty

Kenneth Craig

Elizabeth Crane

Kathryn Dindia

Dina Dosmukhambetova

Andrew Elliot

Eric Everhart

Sally Farley

Jose-Miguel Fernandez-Dols

Pierre Feyereisen

Agneta Fischer

Brandi Frisby

Jennifer Gerwing

Pierre Gosselin

Vladas Griskevicius
Laura Guerrero

Schlomo Hareli

Ginni Harrigan

$\mathrm{Pa} \mathrm{Her}$

Matthew Hertenstein

Jari Hietanen

Judith Holler

Terrence Horgan

Autumn Hostetter

Roland Imhoff

Derek Isaacowitz

Roxane Itier

Lucy Johnston

Benedict Jones

Masanori Kimura

Namkje Koudenburg

Robert Krauss

Jessica Lakin

Heather Lench

Justin Matthews

Christopher Nave

Louis Penner

Frank Pollick

Drew Rendall

Nicholas Rule

Guiliana Salvato

Jennifer Samp
Sascha Schwarz

Atsushi Senju

Ryne Sherman

Leanne ten Brinke

Pascal Thibault

Erin Tone

Job van der Schalk

Peter Weyers

Matthias Weiser

Miron Zuckerman 